$10.1117 / 2.1201306 .004958$

\title{
Extreme UV interference lithography: progress and prospects
}

\section{Yasin Ekinci}

Interference lithography using extreme-UV light is a powerful, lowcost method with applications in both ind ustrial and academic research, including photoresist screening and high-resolution patterning.

Extreme-UV (EUV) lithography using light of wavelength $13.5 \mathrm{~nm}$ is considered the leading technology option for future nodes (a reference to the smallest feature size) of high-volume semiconductor manufacturing. ${ }^{1,2}$ In addition to the main challenge of developing high-power plasma sources, the performance of EUV resists-defined by the trade-off in resolution (half-pitch, HP), sensitivity (dose), and line-edge roughness-is one of the key factors for the cost-effectiveness of this technique. Researchers have invested intensive global effort in the development of resists using available projection tools. However, the resolution of these tools is limited due to the numerical aperture of the projection optics. For the future of EUV lithography, it is crucial to evaluate and eventually demonstrate the extendibility of the different resist approaches toward further technology nodes to have sufficient lead time to enable the commercialization of these materials.

Interference lithography (IL) is a simple and low-cost method of creating aerial images without the limitations of projection optics. In this technique, the interference of two or more coherent beams generates a periodic aerial image on the wafer plane. EUV-IL combines the advantages of IL and the short wavelength of EUV. The EUV-IL tool installed at the Swiss Light Source at the Paul Scherrer Institute in Switzerland uses an undulator source to coherently and homogeneously illuminate the transmission grating masks. The gratings are written with electronbeam lithography on silicon nitride membranes. The diffracted beams of different gratings with equal period interfere and create a sinusoidal aerial image: see Figure 1 The period of the interference pattern is half the period of the gratings if we use the first-order diffracted beams for interference. Unless a particular

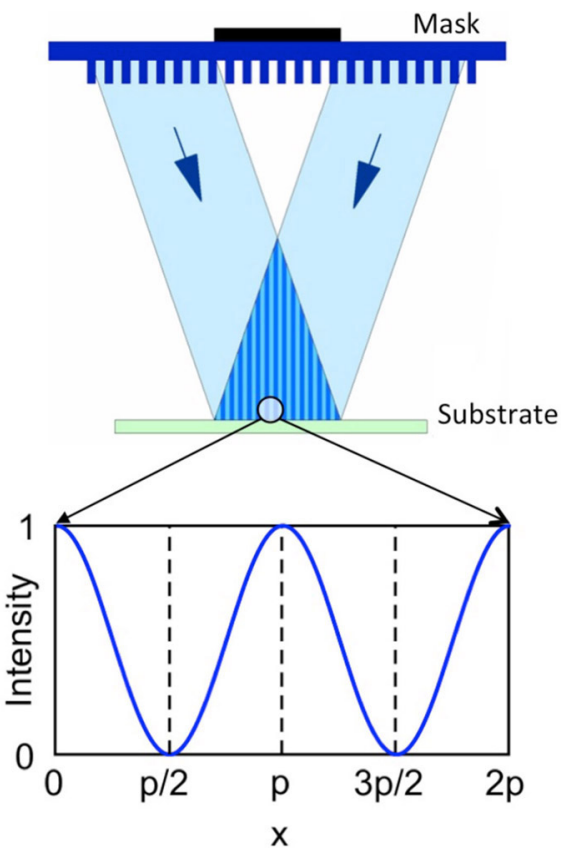

Figure 1. Interference scheme using diffraction gratings. We illuminate transmission gratings on a thin membrane using a spatially coherent beam. Diffracted beams interfere on the wafer plane creating a sinusoidal aerial image. All areas outside the gratings on the mask are coated with a thick polymer film, which completely absorbs the zeroorder beam. p: Period of aerial image modulation.

application requires a large area of a single pattern, the masks consist of several grating pairs enabling versatile nanostructures or different HPs at a single exposure. The EUV-IL technique has infinite depth of focus, i.e., the aerial image is insensitive to gap variations, and the patterned area varies from $50 \times 50 \mu \mathrm{m}^{2}$ to $5 \times 5 \mathrm{~mm}^{2}$ depending on the mask design. 


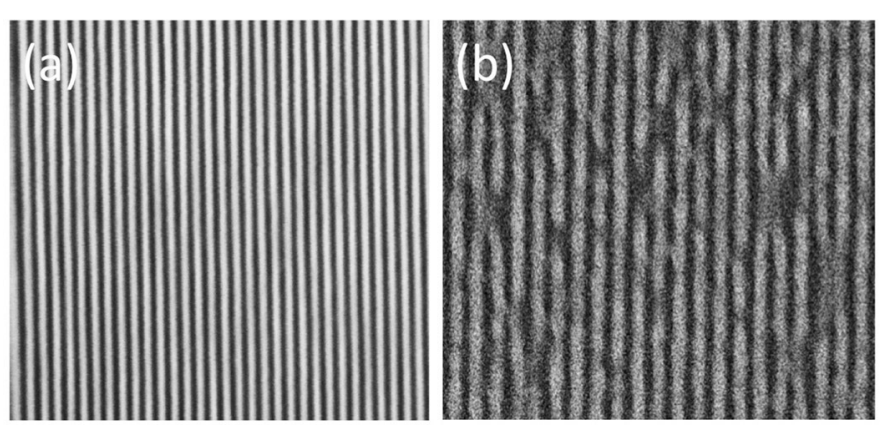

Figure 2. Scanning electron microscopy images of the highest resolutions obtained with (a) an extreme UV-sensitive inorganic resist down to $8 \mathrm{~nm}$ half-pitch and $(b)$ a chemically amplified resist achieving a resolution of $12 \mathrm{~nm}$ half-pitch with partial pattern collapse.

In the last few years, the EUV-IL device at the Swiss Light Source has emerged as a promising tool for academic and industrial research due to its high resolution and well-defined aerial image, high throughput, and large-area capabilities. Its industrial applications mainly involve EUV resist testing. ${ }^{3-6}$ It also helps academic researchers by proving nanostructures for various applications such as directed self-assembly, nanomagnetism, nanoplasmonics, and nanoimprint templates. ${ }^{7}$ Further, it demonstrated resolutions of 12.5, 11, and 8nm HP in 2007, 2009, and 2012, respectively. ${ }^{5-10}$ Recently, we showed 7nm HP resolution using a hydrogen silsesquioxane resist. However, this is a slow resist and, therefore, not feasible for EUV lithography.

For this technique, the state-of-the-art in EUV resists is very promising, although significant progress is still needed. Figure 2 shows an EUV-sensitive inorganic resist with a resolution of 8nm HP. Hafnium-based inorganic resists show very good performance with relatively low dose due to the high absorption of hafnium at EUV wavelengths. 5 , 6 For high sensitivity, chemically amplified resists are the most promising materials. With these, we have achieved resolved patterns down to 12nm HP: see Figure 2 With decreasing HP, however, pattern collapse becomes a crucial issue for all materials, and organic ones are more prone to such problems. Thus, pattern collapse will be a major problem for future technology nodes that will require researchers to apply mitigation techniques such as supercritical drying or special development recipes.

In addition to EUV, we have shown patterning with interference lithography at other wavelengths such as 6.5nm (beyond EUV) and in the soft x-ray range up to the oxygen absorption edge. ${ }^{11}$ Moreover, the type of patterns that can be fabricated with EUV-IL is not limited to line or space patterns. Using multiple beams and precise control of the phase of individual beams or achromatic Talbot lithography with single gratings, we have demonstrated versatile nanostructures, including hole, dot, and ring arrays, non-diffracting Bessel beams, Penrose patterns, and kagome patterns. ${ }^{12-16}$

As these achievements show, EUV-IL is a powerful method for high-resolution lithography as well as for evaluating EUV resists for future technology nodes. We have shown that EUV photons can resolve down to $7 \mathrm{~nm} \mathrm{HP}$, demonstrating that there are no fundamental limitations in terms of photochemistry and materials science. New possibilities emerge with patterning in the sub$10 \mathrm{~nm}$ range, a length scale that has been the domain of chemical synthesis and bottom-up approaches to study quantum effects and others. Now, top-down methods like EUV-IL will enable the study of these interesting systems with better control and longrange order. These are the topics of our future work.

\section{Author Information}

\section{Yasin Ekinci \\ Paul Scherrer Institute (PSI) \\ Villigen PSI, Switzerland}

Yasin Ekinci, a senior scientist, is the manager of the XIL-II (X-ray Interference Lithography) beamline at the Swiss Light Source and leads the nano-optics group in the Laboratory for Microand Nanotechnology. His research interests are EUV interference lithography and nanostructures for optical methods and applications.

\section{References}

1. http://www.itrs.net/International Technology Roadmap for Semiconductors. Accessed 19 June 2013.

2. G. Tallents, E. Wagenaars, and G. Pert, Optical lithography: lithography at EUV wavelengths, Nat. Photon. 4, p. 809, 2009.

3. R. Gronheid, H. H. Solak, Y. Ekinci, A. Jouve, and F. Van Roey, Characterization of extreme ultraviolet resists with interference lithography, Microelectron. Eng. 67-68, p. 1103, 2006.

4. A. M. Goethals, R. Gronheid, F. Van Roey, H. H. Solak, and Y. Ekinci, Progress in EUV resist performance, J. Photopolym. Sci. Technol. 19, p. 501, 2006.

5. Y. Ekinci, M. Vockenhuber, B. Terhalle, M. Hojeij, L. Wang, and T. R. Younkin, Evaluation of resist performance with EUV interference lithography for sub-22nm patterning, Proc. SPIE 8322, p. 83220W, 2012. doi:10.1117/12.916541

6. Y. Ekinci, M. Vockenhuber, M. Hojeij, L. Wang, and N. M. Mojarad, Evaluation of EUV resist performance with interference lithography towards $11 \mathrm{~nm}$ half-pitch and beyond, Proc. SPIE 8679, p. 867910, 2013. doi:10.1117/12.2011533

7. V. Auzelyte, C. Dais, P. Farquet, D. Grützmacher, L. J. Heyderman, F. Luo, S. Olliges, et al., Extreme ultraviolet interference lithography at the Paul Scherrer Institut, J. Micro/Nanolith. MEMS MOEMS 8 (2), p. 021204, 2009. doi:10.1117/1.3116559

8. H. H. Solak, Y. Ekinci, P. Käser, and S. Park, Photon-beam lithography reaches 12.5nm half-pitch resolution, J. Vac. Sci. Technol. B 25, p. 91, 2007.

9. B. Päivänranta, A. Langner, E. Kirk, C. David, and Y. Ekinci, Sub-10nm patterning using EUV interference lithography, Nanotechnol. 22, p. 375302, 2011.

10. Y. Ekinci, H. H. Solak, C. Padeste, J. Gobrecht, M. P. Stoykovich, and P. F. Nealey, 20nm line/space patterns in HSQ fabricated by EUV interference lithography, Microelectron. Eng. 84, p. 700, 2007. 
11. N. Mojarad, M. Vockenhuber, L. Wang, B. Terhalle, and Y. Ekinci, Patterning at 6.5nm wavelength using interference lithography, Proc. SPIE 8679, p. 867924, 2013. doi:10.1117/12.2011556

12. L. Wang, B. Terhalle, V. A. Guzenko, A. Farhan, M. Hojeij, and Y. Ekinci, Generation of high-resolution kagome lattice structures using extreme ultraviolet interference lithography, Appl. Phys. Lett. 101, p. 093104, 2012.

13. A. Langner, B. Päivänranta, B. Terhalle, and Y. Ekinci, Fabrication of quasiperiodic nanostructures with EUV interference lithography, Nanotechnol. 23, p. 105303, 2012.

14. B. Terhalle, A. Langner, B. Päivänranta, C. David, and Y. Ekinci, Generation of EUV vortex beams using computer generated holograms, Opt. Lett. 36, p. 4143, 2011

15. B. Terhalle, A. Langner, B. Päivänranta, and Y. Ekinci, Advanced holographic methods in extreme ultraviolet interference lithography, Proc. SPIE 8192, p. $81020 \mathrm{~V}$, 2011. doi:10.1117/12.893733

16. H. H. Solak and Y. Ekinci, Achromatic spatial frequency multiplication: a method for production of nanometer-scale periodic structures, J. Vac. Sci. Technol. B 23, p. 2705, 2005. 